\title{
Effects of concurrent arithmetical and syntactic complexity on self-paced reaction times and eye fixations
}

\author{
William S. Evans • David Caplan • Gloria Waters
}

Published online: 29 September 2011

(C) Psychonomic Society, Inc. 2011

\begin{abstract}
Two dual-task experiments (replications of Experiments 1 and 2 in Fedorenko, Gibson, \& Rohde, Journal of Memory and Language, 56, 246-269 2007) were conducted to determine whether syntactic and arithmetical operations share working memory resources. Subjects read object- or subject-extracted relative clause sentences phrase by phrase in a self-paced task while simultaneously adding or subtracting numbers. Experiment 2 measured eye fixations as well as self-paced reaction times. In both experiments, there were main effects of syntax and of mathematical operation on self-paced reading times, but no interaction of the two. In the Experiment 2 eye-tracking results, there were main effects of syntax on first-pass reading time and total reading time and an interaction between syntax and math in total reading time on the noun phrase within the relative clause. The findings point to differences in the ways individuals process sentences under these dual-task conditions, as compared with viewing sentences during "normal" reading conditions, and do not support the view that arithmetical and syntactic integration operations share a working memory system.
\end{abstract}

Electronic supplementary material The online version of this article (doi:10.3758/s13423-011-0159-9) contains supplementary material, which is available to authorized users.

W. S. Evans $\cdot$ G. Waters

Department of Speech, Language, and Hearing Sciences,

Sargent College, Boston University,

Boston, MA, USA

D. Caplan $(\varangle)$

Neuropsychology Laboratory, Department of Neurology,

Massachusetts General Hospital,

Boston, MA, USA

e-mail: dcaplan@partners.org
Keywords Dual-task performance $\cdot$ Eye movements and reading $\cdot$ Working memory $\cdot$ Language comprehension

\section{Introduction}

The issue of whether language processing utilizes a verbal working memory system that is employed in other verbally mediated tasks (e.g., Fedorenko, Gibson, \& Rohde, 2007; King \& Just, 1991) or, instead, relies on a special set of resources dedicated specifically to language processing (e.g., Caplan \& Waters, 1999) is unresolved. Fedorenko et al. examined the domain specificity of the working memory system responsible for one aspect of language - syntactic processing -in a unique series of self-paced dual-task experiments. In one set of experiments, subjects performed reading and arithmetic tasks simultaneously, while in another the reading task was paired with spatial tasks. In both series of experiments, the difficulty of each task was varied in $2 \times 2$ designs. Sentences containing subject-extracted relative clauses (SRCs) or object-extracted relative clauses (ORCs) were presented phrase by phrase in self-paced reading, with an arithmetic or spatial computation appearing simultaneously with each phrase (Fig. 1). Fedorenko et al. found a superadditive interaction when the sentence and arithmetic tasks were combined, with response times in the hardsentence/hard-math condition being disproportionately long when the relative clause was viewed. In contrast, they did not find a superadditive interaction when they paired the sentence task with a spatial integration task. On the basis of additive factors logic (Sternberg, 1969), they argued that reading and arithmetical operations rely on a common pool of working memory resources, while reading and spatial processing rely on separate pools. Fedorenko et al. argued that they obtained a superadditive interaction between 


\begin{tabular}{|c|c|c|c|c|}
\hline Time 1: & $\begin{array}{l}12 \\
\text { The janitor }\end{array}$ & 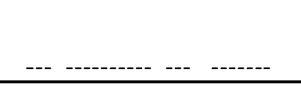 & - & - - \\
\hline Time 2: & 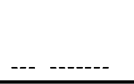 & $\begin{array}{l}+4 \\
\text { who frustrated the plumber }\end{array}$ & $-\cdots$ & \\
\hline Time 3: & --- ------- & 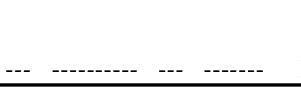 & $\begin{array}{l}+5 \\
\text { lost the key }\end{array}$ & \\
\hline ime 4: & -- ------- & $\begin{array}{llll}--- & --------- & --- & ------- \\
\end{array}$ & --- --- --- & $\begin{array}{l}+4 \\
\text { on the stre }\end{array}$ \\
\hline
\end{tabular}

Fig. 1 Depiction of self-paced dual-task presentation from Fedorenko et al. (2007), Experiment 1

syntactic and arithmetical complexity because both tasks required both retrieval and integration of information, unlike in previous dual-task studies in which the concurrent memory task (immediate serial recall) required only retrieval (Caplan \& Waters, 1999; Waters, Caplan, \& Rochon, 1995).

The present study constitutes an effort to replicate Fedorenko et al. (2007) results (Experiment 1) and to examine the effect of combining sentence and arithmetic processing more closely by measuring eye fixations (Experiment 2), which potentially provide a more finegrained measure of the different aspects of processing involved in this type of task.

\section{Experiment 1}

\section{Method}

Subjects Fifty subjects were recruited from Boston University (BU). All were native speakers of English between the ages of 18 and 30 years, possessed normal/corrected-to-normal vision, and had no history of neurological injury or impairment.

Materials and procedure The materials and procedures were identical to those in Experiment 1 in Fedorenko et al. (2007). The experiment was presented using a self-paced reading, phrase-by-phrase design with a moving-windows display. SRCs ("The janitor/who frustrated the plumber/lost the key/on the street.") and ORCs ("The janitor/who the plumber frustrated/lost the key/on the street.") sentences were displayed region by region, with a number presented simultaneously directly above each region. In the easy arithmetic condition, the first term was between 1 and 10 , and successive addends were between 1 and 3 . In the hard arithmetic condition, the first term was between 11 and 20, and successive addends were between 4 and 6 . Subjects were required to answer two questions about the sentence and to compute the sum of the series of numbers. Testing took place on a Macintosh laptop computer using Linger (http://tedlab.mit.edu/ dr/Linger/) and used the Linger script written by Fedorenko and colleagues. Buttonpress reaction times were recorded for each sentence region.

\section{Results}

Accuracy and reaction time data were analyzed using mixed logistic regression models (Jaeger, 2008) and linear mixed effects models (Baayen, Davidson, \& Bates, 2008), respectively, both with crossed random subject and item effects. An initial model that included fixed effects of math and syntax, a math $\times$ syntax interaction, and random subject and item slopes was compared with models in which the fixed effects were sequentially removed on the basis of significance level and log likelihood comparisons. For the sake of brevity, only the most highly valued model is reported for each analysis.

Accuracy Subjects were $90 \%$ accurate on arithmetic questions and $78 \%$ accurate on comprehension questions, overall (Table 1). There were main effects of arithmetic complexity on math question accuracy, such that harder arithmetic resulted in less accurate responses, and of syntax on comprehension question accuracy, with less accurate responses in the ORC condition (Table 2). There were no interaction effects. The arithmetic and comprehension question accuracy from Experiment 1 was also compared with the original Experiment 1 data from Fedorenko et al. (2007) (Table 1; Supplementary Material: Table S1), and the groups did not significantly differ.

Reaction time Reaction times more than 3 standard deviations from each condition were discarded (affecting 1.9\% of the data, overall). Remaining reaction times were analyzed separately by region, using linear mixed effects models. ${ }^{1}$ Analyses were conducted on all trials regardless of accuracy, as in Fedorenko et al. (2007). Table 3 presents mean reading times by region and condition (with comparisons with those in Fedorenko et al., 2007), and Table 4 presents model results for each condition and region. Analysis of the critical region 2 containing the SRC/ORC contrast revealed main effects for both math and syntax, such that the harder conditions in each instance corresponded to longer reaction times, but there was no interaction ${ }^{2}(p=.32$ in the initial model). Examination of the other regions revealed

\footnotetext{
${ }^{1}$ Fedorenko et al. (2007) used residuals to adjust for region lengths and individual subjects' reading rates. Mixed effects analysis, with a length factor when needed, adjusts for these factors by generating crossed random intercepts for each subject and item (Baayen et al., 2008).

${ }^{2}$ There was sufficient power to detect an interaction effect in this model given the effect sizes seen in Experiment 1 in Fedorenko et al. (2007) and our sample size (power $=.985$ ). Power analysis was conducted using online Java software (Lenth 2009).
} 
Table 1 Present Experiment 1 question accuracy compared with results of Experiment 1 in Fedorenko et al. (2007): Mean accuracy by condition (with standard errors in parentheses)

\begin{tabular}{lll}
\hline & Easy Syntax (SRC) & Hard Syntax (ORC) \\
\hline $\begin{array}{ll}\text { Mean Accuracy by Condition for Present Experiment } 1 \\
\text { Math Accuracy }\end{array}$ & \\
Easy math (small addends) & $92.8 \%(1.3)$ & $92.3 \%(1.4)$ \\
Hard math (large addends) & $86.3 \%(1.7)$ & $88.0 \%(1.6)$ \\
Comprehension Question Accuracy & & \\
Easy math (small addends) & $81.8 \%(1.4)$ & $76.3 \%(1.5)$ \\
Hard math (large addends) & $78.9 \%(1.4)$ & $75.1 \%(1.5)$ \\
Mean Accuracy by Condition for Experiment & 1 in Fedorenko et al. (2007) \\
Math Accuracy & $93.5 \%(1.7)$ & $89.8 \%(1.8)$ \\
Easy math (small addends) & $86.7 \%(2.4)$ & $87.8 \%(2)$ \\
Hard math (large addends) & & $78.8 \%(2.7)$ \\
Comprehension Question Accuracy & $85.8 \%(2.1)$ & $77.0 \%(2.1)$ \\
Easy math (small addends) & $80.2 \%(2.4)$ & \\
Hard math (large addends) &
\end{tabular}

main effects for math in regions 1,3 , and 4, such that trials containing hard arithmetic took longer.

Raw reaction times from region 2 in this experiment were compared with the original data from Fedorenko et al. (2007) (Supplementary Material: Tables S2, S3). There were interactions between math and population and between syntax and population, such that our subjects took longer than those in Fedorenko et al. in the difficult conditions. There was also an overall interaction between math and syntax, as found in the Fedorenko et al. data alone. The three-way interaction of population, math, and syntax was not significant.

In order to determine whether individual differences in processing efficiency played a role in determining the size of the interaction effect, the correlation between the by-subject reading time adjustment and the interaction terms was calculated in the combined group (Supplementary Material: Table S4). The correlation was not significant $(r=.062)$.

Table 2 Mixed logistic regression analysis of question accuracy in Experiment 1: Effects of syntax (reference level: SRC) and math (reference level: small addends) on $\log$ (odds) accuracy. Math $\times$ syntax interaction terms are dropped from final models due to lack of significance $(z \mathrm{~s}<1)$. All models contain crossed random subject/item intercepts

\begin{tabular}{lcccc}
\hline & Estimate (log odds) & $S E$ & $z$ Value & $p$ Value \\
\hline Math Accuracy & & & & \\
Intercept & 2.66 & 0.19 & 14.15 & \\
Syntax effect & 0.07 & 0.17 & 0.42 & .67 \\
Math effect & 0.62 & 0.18 & 3.56 & $<.001$ \\
Comprehension Question Accuracy & & & \\
Intercept & 0.62 & 0.14 & 11.86 & \\
Syntax effect & 0.29 & 0.09 & 3.25 & .001 \\
Math effect & 0.14 & 0.09 & 1.53 & .13 \\
\hline
\end{tabular}

\section{Discussion}

Although we replicated the main effects of arithmetical and syntactic complexity on answering questions and self-paced reaction times reported in Fedorenko et al. (2007), we failed to observe their reported interaction of these factors in region 2 in our experiment. The results suggest that there are individual differences in the processes that generated the interaction in Fedorenko et al. (Experiment 1). These individual differences do not appear to be due to individual differences in a shared working memory system, because there was no association between individual processing efficiency, as measured by overall processing speed, and the size of the interaction effect.

Although we did not replicate Fedorenko et al.'s (2007) result, both their result and the finding of an interaction of math and syntax in the critical segment in the analysis combining the two populations may be taken as evidence of an interaction between math and syntax under these dual-task conditions. Experiment 2 addressed the question of whether the interaction would arise with another group of subjects taken from the BU population when a second, similar design was used (Fedorenko et al., 2007, Experiment 2) and further explored the interaction by measuring eye fixations.

\section{Experiment 2}

Method

Subjects Forty subjects were recruited from BU, using the same selection criterion as in Experiment 1.

Materials Materials were taken from Experiment 2 in Fedorenko et al. (2007). Sentential materials and fillers 
Table 3 Present Experiment 1 mean self-paced reading times (in raw milliseconds) compared with results of Experiment 1 in Fedorenko et al. (2007), separately by region and condition. Arithmetic conditions, small addends versus large addends; syntax conditions, subject-extracted relative clause versus object-extracted relative clause (SRC/ORC). Critical region 2 is shown in bold. Standard errors in parentheses

\begin{tabular}{|c|c|c|c|c|}
\hline Self-Paced Reading Time (ms) & Region 1 & Region 2 & Region 3 & Region 4 \\
\hline \multicolumn{5}{|l|}{ Present Experiment 1} \\
\hline Small addends/SRC & $1,584(40)$ & $2,608(66)$ & $2,413(55)$ & $1,976(52)$ \\
\hline Large addends/SRC & $1,835(60)$ & $3,156(81)$ & $3,080(89)$ & $2,393(62)$ \\
\hline Small addends/ORC & $1,634(47)$ & $3,137(87)$ & $2,485(58)$ & $1,827(47)$ \\
\hline Large addends/ORC & $1,679(47)$ & $3,866(109)$ & $2,907(69)$ & $2,391(62)$ \\
\hline \multicolumn{5}{|c|}{ Experiment 1 in Fedorenko et al. (2007) } \\
\hline Small addends/SRC & $1,444(35)$ & $2,411(56)$ & $2,294(54)$ & $1,675(36)$ \\
\hline Large addends/SRC & $1,580(40)$ & $2,713(63)$ & $2,631(68)$ & $2,199(55)$ \\
\hline Small addends/ORC & $1,473(42)$ & $2,689(63)$ & $2,260(56)$ & $1,700(43)$ \\
\hline Large addends/ORC & $1,625(45)$ & $3,177(84)$ & $2,703(70)$ & $2,263(64)$ \\
\hline
\end{tabular}

were identical to those used in Experiment 1. Arithmetical complexity was manipulated by varying the type of operation involved (first addition term between 30 and 50; first subtraction term between 40 and 60; subsequent addends/subtrahends between 3 and 6).

Design and procedure The procedure followed the Experiment 1 methodology, with the following change: Subjects were tested using an Eyelink II head-mounted eye-tracking system (SR Research, Ltd), and the number and the phrase in each segment were separated by $8 \mathrm{~cm}$ (approximately $8.5^{\circ}$ of visual field) to eliminate parafoveal information. Arithmetic and comprehension question accuracy (via computer keyboard), response times for each region (via USB response pad), and fixation data were recorded.

Results

Accuracy Subjects were $83 \%$ accurate on arithmetic questions and $71 \%$ accurate on comprehension questions, overall. Accuracy data (Tables 5 and 6) were

Table 4 Linear mixed effects analysis of reaction time by region in Experiment 1: Effects of syntax (reference level: SRC) and math (reference level: small addends) on raw reaction times in milliseconds.

Math $\times$ syntax interaction terms are dropped from all final models when $p \mathrm{MCMC}>.1$ in the initial model. All models contain crossed random subject/item intercepts

\begin{tabular}{|c|c|c|c|c|}
\hline & Estimate (ms) & $S E$ & $t$ Value & $p$ Value (MCMC) \\
\hline \multicolumn{5}{|c|}{ Region 1 (“The janitor") } \\
\hline Intercept & $1,626.38$ & 96.24 & 16.90 & \\
\hline Syntax effect & 40.10 & 58.14 & 0.69 & .494 \\
\hline Math effect & 199.77 & 58.22 & 3.43 & $<.001$ \\
\hline Math $\times$ syntax & 143.02 & 82.03 & 1.74 & .089 \\
\hline \multicolumn{5}{|c|}{ Region 2 ("who frustrated the plumber/who the plumber frustrated") } \\
\hline Intercept & $2,618.20$ & 163.25 & 16.04 & \\
\hline Syntax effect & 581.20 & 69.27 & 8.39 & $<.001$ \\
\hline Math effect & 584.97 & 70.31 & 8.32 & $<.001$ \\
\hline \multicolumn{5}{|c|}{ Region 3 ("lost the key") } \\
\hline Intercept & $2,481.36$ & 125.71 & 19.74 & \\
\hline Syntax effect & 18.34 & 59.07 & 0.31 & .755 \\
\hline Math effect & 561.56 & 59.80 & 9.39 & $<.001$ \\
\hline \multicolumn{5}{|c|}{ Region 4 ("on the street") } \\
\hline Intercept & $1,960.25$ & 93.41 & 20.99 & \\
\hline Syntax effect & 79.58 & 50.50 & 1.58 & .120 \\
\hline Math effect & 511.98 & 50.50 & 10.14 & $<.001$ \\
\hline
\end{tabular}


Table 5 Mean Experiment 2 question accuracy by condition with standard errors in parentheses

\begin{tabular}{lll}
\hline & Easy Syntax (SRC) & Hard Syntax (ORC) \\
\hline Arithmetic Accuracy & & \\
Easy math (addition) & $84.0 \%(1.3)$ & $80.1 \%(1.2)$ \\
Hard math (subtraction) & $83.6 \%(1.2)$ & $84.0 \%(1.2)$ \\
Comprehension Question Accuracy & & $69.5 \%(1.1)$ \\
Easy math (addition) & $75.4 \%(1.0)$ & $68.6 \%(1.1)$ \\
Hard math (subtraction) & $73.0 \%(1.1)$ & \\
\hline
\end{tabular}

analyzed as in Experiment 1. A main effect of syntactic complexity on math question accuracy was found, such that responses were less accurate in the ORC condition. There were no other significant effects for either question type.

Reaction time Self-paced reaction times (Tables 7 and 8) were analyzed as in Experiment 1. Of the data, $1.7 \%$ were discarded as outliers. Analysis of the critical region 2 containing the SRC/ORC contrast revealed main effects for both math and syntax, such that the harder conditions in each instance corresponded to longer reaction times, but there was no interaction ( $p$ value $=.73$ in the initial model). Examination of the other regions revealed main effects for math in regions 3 and 4, such that trials with subtraction took longer.

Eye-tracking analyses Fixations on regions before stimuli appeared and after they disappeared in the self-paced moving window were removed $(2.5 \%$ and $3.8 \%$ of total fixations, respectively). For the sake of brevity, analyses will focus on region 2, with interest areas consisting of the relative pronoun ("who"), the noun phrase (NP; "the plumber"), the verb ("frustrated"), and the number appear-

Table 6 Mixed logistic regression analysis of question accuracy in Experiment 2: Effects of syntax (reference level: SRC) and math (reference level: addition) on $\log$ (odds) accuracy. Models contain crossed random subject/item intercepts and random slopes for math $\times$ syntax interactions

\begin{tabular}{lcccc}
\hline & Estimate (log odds) & $S E$ & $z$ Value & $p$ Value \\
\hline Math Accuracy & & & & \\
Intercept & 2.95 & 0.40 & 7.38 & \\
Syntax effect & 0.94 & 0.40 & 2.35 & .019 \\
Math effect & 0.02 & 0.55 & 0.03 & .978 \\
Math $\times$ syntax & 0.77 & 0.68 & 1.13 & .257 \\
Comprehension Question & Accuracy & & & \\
Intercept & 1.32 & 0.22 & 5.89 & \\
Syntax effect & 0.40 & 0.25 & 1.60 & .110 \\
Math effect & 0.14 & 0.21 & 0.68 & .497 \\
Math $\times$ syntax & 0.12 & 0.31 & 0.37 & .713 \\
\hline
\end{tabular}

ing in this region ("number 2"). Three reading time measures were calculated for these interest areas: first-pass reading time (the sum duration of the first fixation plus all subsequent fixations until the subject left the interest area), total reading time (the sum duration of all fixations on the interest area), and second-pass reading time (the difference between first-pass and total reading times). Mean reading times by condition, interest area, and reading measure were calculated (Table 9), and data were analyzed using linear mixed effects models, as above (Table 10). Second-pass reading times produced the same results as total reading times and are not reported.

For the number in region 2, there was a main effect of math on total reading time, such that subjects took longer on trials with subtraction. There were no other effects in either first-pass or total reading time.

For the relative pronoun, there was a main effect for syntax on both first-pass and total reading time, such that subjects took longer in the ORC condition. There were no math effects and no interaction.

For the relative clause verb, there were no syntax effects on first-pass reading time, and total reading time was longer on the SRC verb than the ORC verb. There were no math effects and no interaction.

For the NP, there were main effects of both syntax and math on first-pass reading time, with the hard conditions (subtraction, ORC) taking longer in each case. There was also a main effect of syntax and an interaction between math and syntax in total reading time, with the ORC/hard arithmetic condition taking the longest.

In order to make certain that first-pass reading times for the relative clause were influenced by demands made by concurrent arithmetic, we analyzed the subset of first-pass reading times following a fixation on the number in region $2(94 \%$ of trials for the noun and verb interest areas and $80 \%$ of trials for the relative pronoun). In this set of analyses, the main effect of syntax on first-pass reading time for the NP was reduced to a trend $(p=.1)$, but there were no other changes in significance for any other factor or interest area.

To assess the effect of changing fixations from math to syntax, we analyzed total reading time following fixations 
Table 7 Experiment 2 mean self-paced reading times (in raw milliseconds), by interest area and condition. Critical region 2 is shown in bold. Standard errors in parentheses

\begin{tabular}{lllll}
\hline Self-Paced Reading Time (ms) & Region 1 & Region 2 & Region 3 & Region 4 \\
\hline Addition/SRC & $2,130(46)$ & $\mathbf{3 , 4 7 6 ( 7 9 )}$ & $3,318(74)$ & $2,767(66)$ \\
Subtraction/SRC & $2,172(50)$ & $\mathbf{3 , 7 5 3}(\mathbf{8 9})$ & $3,566(87)$ & $3,087(76)$ \\
Addition/ORC & $2,114(46)$ & $\mathbf{3 , 8 2 1}(\mathbf{9 7})$ & $3,350(79)$ & $2,836(69)$ \\
Subtraction/ORC & $2,154(48)$ & $\mathbf{4 , 0 6 1 ( 9 9 )}$ & $3,726(90)$ & $3,069(79)$ \\
\hline
\end{tabular}

on the same and different type of material ${ }^{3}$ (syntax, math) (Supplementary Materials: Tables S5 and S6). For fixations on the NP following fixations on the number, there were no main effects or interactions of arithmetical or syntactic complexity. For fixations on the NP following fixations on other sentence segments, there were no main effects, but there was a significant superadditive interaction of arithmetical and syntactic complexity.

To determine whether the interaction of syntactic and arithmetic complexity in total reading times for the NP was due to subjects' fixating the NP after the verb in ORCs (i.e., to initially skipping the ORC NP), we analyzed the proportion of first fixations on the verb and NP of each sentence type in each condition. Of the first fixations, 99\% fell on the NP before the verb in ORCs.

Finally, we also analyzed results by positions - position 1 consisting of the verb in the SRC condition and the NP in the ORC condition, and position 2 consisting of the NP in the SRC condition and the verb in the ORC condition (Supplementary Materials: Table S7). Because of differences in lexical items in these positions in SRC and ORC sentences, interest area word length and average log frequency (SUBTLEXus database; Brysbaert \& New, 2009) were included as factors in these models. There were main effects of math and syntax on first-pass reading time and on total reading time, such that the ORC and subtraction conditions took longer. There was an interaction between position and math for total reading time (and a trend in the first-pass measure), such that reading times were disproportionately long in the position 1/subtraction condition, and between position and syntax in total reading time, such that reading times were disproportionately long in the position 1/ORC condition. The three-way interaction between math, syntax, and position was not significant ( $p>.1$ in the initial model).

\section{Discussion}

We replicated the main effects of arithmetical and syntactic complexity on self-paced reading times reported in Fedorenko et al. (2007) but again failed to find an interaction of these

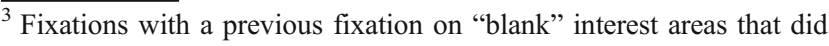
not contain informative content (dashes) or on noninterest area locations were dropped from the analysis, affecting $53 \%$ of the trials.
}

factors in region 2 in our experiment. Eye fixations showed some expected effects of complexity (we discuss unexpected results below). There was an interaction of math and syntax in total reading time for the NP. The interaction itself points to some process or resource that affects both the syntactic and arithmetic tasks. The fact that it was found only when subjects had immediately previously fixated on the sentence material indicates that it was not due to costs of shifting visual focus (and, presumably, attention) from one input and task to the other. The fact that it was found following fixations on the sentence, and not on the number, is also consistent with its being due to a shared resource: Inspecting the number might have allowed subjects to complete arithmetic processing or to reinforce the encoded intermediate product of computation, thereby reducing concurrent memory demand while they accomplished the sentence task and eliminating the interaction of arithmetic and sentence processing. The remaining, critical question is what process(es) does this shared resource support.

The locus and timing of the interaction differ from the locus and timing of the effect of syntactic complexity on eye fixations for reading sentences in isolation. Effects of the ORC structure at the NP consist of more regressions and (consequently) longer go-past reading times (Staub, 2010) and have been attributed to surprisal (Levy, 2008; Staub, 2010). Effects of the ORC structure also are seen in the form of longer first-pass reading times for the verb (Staub, 2010), which have been attributed to integration of information retrieved from memory with the current input. There was no interaction of syntax and arithmetic for this constituent in this study. Thus, neither the location nor the timing of the observed interaction supports Fedorenko et al.'s (2007) claim that it results from a working memory system that is common to syntactic and arithmetical operations. However, features of the eye fixations in this study suggest that it is risky to base interpretations of eye fixations here on results in normal reading. We take this up in the General Discussion section.

\section{General discussion}

The self-paced reaction time results in these studies differ from those in Fedorenko et al. (2007), in that we did not 
Table 8 Linear mixed effects analysis of reaction time by region in Experiment 2: Effects of syntax (reference level: SRC) and math (reference level: addition) on raw reaction times in milliseconds. Math $\times$ syntax interaction terms are dropped from all final models when $p \mathrm{MCMC}>.1$ in the initial model. All models contain crossed random subject/item intercepts

\begin{tabular}{lcccc}
\hline & Estimate (ms) & $S E$ & $t$ Value & $p$ Value (MCMC) \\
\hline Region 1 ("The janitor") & & & & \\
Intercept & $2,149.10$ & 97.77 & 21.98 & .550 \\
Syntax effect & 21.18 & 34.54 & 0.61 & .306 \\
Math effect & 35.84 & 34.54 & 1.04 & \\
Region 2 (SRC: "who frustrated the plumber"/ORC: "who the plumber frustrated") & \\
Intercept & $3,508.94$ & 190.71 & 18.40 & \\
Syntax effect & 324.53 & 66.43 & 4.89 & .001 \\
Math effect & 261.11 & 66.43 & 3.93 & 22.14 \\
Region 3 ("lost the key") & & & & .163 \\
Intercept & $3,310.89$ & & 149.58 & $<.001$ \\
Syntax effect & 93.57 & 67.65 & 1.38 & \\
Math effect & 310.89 & 67.65 & 4.60 & .748 \\
Region 4 ("on the street") & & & & $<.001$ \\
Intercept & $2,817.72$ & 127.63 & 22.08 & 0.29 \\
Syntax effect & 17.86 & 61.10 & 4.46 & \\
Math effect & 272.28 & 61.08 & &
\end{tabular}

find an interaction of syntactic and arithmetic complexity. The differences between the groups do not seem to be due to individual differences in a working memory capacity that is shared by the two tasks, because there was no correlation between how quickly a subject processed the critical segment and the size of his/her interaction effect. Subjects in our Experiment 1 were less efficient than those in Fedorenko et al.'s (showing larger reading time main effects for both tasks) and would, therefore, have been expected to show the interaction if it arose from reliance of the two processes on a common working memory system. These results thus raise doubts about Fedorenko et al.'s conclusion that operations in these tasks rely on a common working memory system.

The eye fixation results showed an interaction of syntactic and arithmetical complexity in total reading times for the relative clause NP. On the basis of the literature on eye fixations in isolated sentences, the time course and location of this interaction are not consistent with its arising from a resource involved in both parsing and interpretation. However, as has been noted, the results showed some features that have not been described in the reading of isolated sentences. Subjects were slow: First-pass reading times on the first element in the relative clause $(\sim 1,000 \mathrm{~ms})$ were approximately double those reported by Staub (2010), and viewing time for region 2 was 3.5-4 s, much longer than is usually seen in self-paced reading times in isolated sentence reading. Subjects fixated the first constituent in the relative clause differently than in normal reading, showing longer total reading times on the verb of SRCs than ORCs and greater effects of complexity in total reading times for the verb of SRC and the NP of ORC sentences. The differences in self-paced reading times and eye fixations in this task and reading of isolated sentences could be due to
Table 9 Experiment 2 Mean first-pass reading times and total reading times (in milliseconds), by relative clause interest area and condition. Arithmetic conditions, addition versus subtraction; syntax conditions, subject-extracted relative clause versus object-extracted relative (SRC/ORC). Standard errors in parentheses

\begin{tabular}{lllll}
\hline & Relative Pro & RC Verb & NP & Number 2 \\
\hline $\begin{array}{l}\text { First-Pass Reading Time } \\
\text { Addition/SRC }\end{array}$ & $388(21)$ & $591(24)$ & $575(21)$ & $717(32)$ \\
Subtraction/SRC & $368(22)$ & $638(26)$ & $612(22)$ & $689(33)$ \\
Addition/ORC & $469(26)$ & $603(24)$ & $623(21)$ & $724(36)$ \\
Subtraction/ORC & $454(25)$ & $608(26)$ & $728(32)$ & $742(35)$ \\
Total Reading Time & & & & \\
Addition/SRC & $416(23)$ & $804(32)$ & $881(32)$ & $1,109(48)$ \\
Subtraction/SRC & $404(26)$ & $855(34)$ & $893(32)$ & $1,221(58)$ \\
Addition/ORC & $510(28)$ & $755(31)$ & $1,052(34)$ & $1,175(53)$ \\
Subtraction/ORC & $507(30)$ & $759(31)$ & $1,233(48)$ & $1,246(59)$ \\
\hline
\end{tabular}


Table 10 Linear mixed effects analyses of eye fixations within region 2 of Experiment 2:

Effects of syntax (reference level: SRC) and math (reference level: addition) on raw first pass, first pass after a fixation on number 2 , and total reading time in milliseconds on relative pronoun, noun phrase, and verb. Math $\times$ syntax interaction terms are dropped from all final models when $p$ MCMC $>.1$ in the initial model. All final models contain crossed random subject/item intercepts

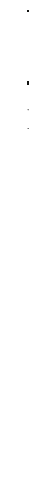

Relative Pronoun ("who")

First-Pass Reading Time

$\begin{array}{lrrrr}\text { Intercept } & 373.35 & 32.00 & 11.67 & \\ \text { Syntax effect } & 95.26 & 21.46 & 4.44 & <.001 \\ \text { Math effect } & 21.23 & 21.42 & 0.99 & 338\end{array}$

21.23

21.42

.338

First-Pass Reading Time After Fixation on Number 2

Intercept

333.52

28.03

11.90

Syntax effect

72.19

21.45

3.37

.001

Math effect

26.16

21.32

1.23

.220

Total Reading Time

Intercept

395.43

34.90

11.33

Syntax effect

110.42

24.61

4.49

$<.001$

Math effect

8.68

24.57

0.35

.720

RC Verb ("frustrated")

First-Pass Reading Time

$\begin{array}{lrrrr}\text { Intercept } & 599.76 & 37.40 & 16.03 & \\ \text { Syntax effect } & 11.90 & 22.35 & 0.53 & .584 \\ \text { Math effect } & 29.27 & 22.34 & 1.31 & .206\end{array}$

First-Pass Reading Time After Fixation on Number 2

Intercept

522.32

31.70

25.85

Math effect

811.97

Total Reading Time

Intercept

$$
77.35
$$

Syntax effect

33.06

NP ("the plumber")

First-Pass Reading Time

Intercept

556.90

Syntax effect

83.14

Math effect

70.69

First-Pass Reading Time After Fixation on Number 2

\section{Intercept}

Syntax effect

Math effect

Total Reading Time

Intercept

Syntax effect

Math effect

Math $\times$ syntax

Number 2

First Pass Reading Time

Intercept
Syntax effect
Math effect
Total reading time
Intercept
Syntax effect
Math effect

527.50
36.57
51.71

879.62
171.62
11.74
168.79

32.13

21.75

21.74

63.24

45.30

45.34

64.09

697.37

32.64

2.96

1102.13

52.38

93.71

57.77

28.65

96.10

45.30

45.29
22.09

22.09
15.98

1.47

1.20

16.29

2.76

1.18

.008

.248

15.52

3.76

$<.001$

3.20

.002

16.42

1.68

.101

2.38

.014

13.91

3.79

$<.001$

0.26

.801

.008

144

232

$$
2.63
$$

8

8

12.07

1.14

.258

0.10

.894

11.47

1.16

2.07 
either of the two major differences between these conditions - the requirement to do a second task, and the disappearance of each segment once the button has been pushed (which makes many normally occurring regressive eye movements uninformative). The results of this study suggest that subjects react to these features of this task both by increasing their exposure to each segment and by allocating eye fixations to segments within the visible regions in ways that differ from those in normal exposure conditions. This affects the interpretation of the interaction seen in the eye fixation data. If subjects' processing of sentences differed from normal reading conditions, it is possible that the interaction in total reading times on the ORC NP reflects competition of parsing and arithmetic for a single set of resources, which is manifest in ways not expected on the basis of effects in normal reading.

One possibility (suggested by an anonymous reviewer) is that subjects often skipped the ORC NP, leading to different aspects of parsing and interpretation being associated with total reading times on this item than are seen in normal reading. However, subjects did not skip the ORC NP, ruling out this possibility. Another possibility is that subjects postponed some syntactic and interpretive processing, such as the integration of successive representations (or probability distributions of representations) that produces surprisal effects, from initial to later fixations. There is no way to directly verify this possibility.

Alternatively, the interaction of math and syntax seen in total fixations on the ORC NP, but not in first-pass reading times on this segment, may reflect reliance of what Caplan and Waters (1999) called "post-interpretive" languagerelated operations and arithmetic on a common resource pool. One such operation might be a late check of the encoded meaning of the phrase against the input for the purpose of later end-of-sentence verification. Traxler, Morris, and Seely (2002; Traxler, Williams, Blozis, \& Morris, 2005) interpreted regressions from the main clause to the sentence-initial NP in ORCs in this way. In a self-paced study, the only informative regressions in ORCs are from the verb to the NP, and these may serve this purpose. Verification of meaning after it has been assigned and reinforcement of what has been encoded in memory are "post-interpretive" verbally mediated operations that Caplan and Waters suggested are supported by a "general" verbal working memory system that could also support arithmetic.

In summary, interactions between concurrent arithmetical and syntactic complexity in self-paced times in dualtask experiments were not observed in our study population. Eye fixations did show such an interaction, but, on the basis of the results in normal reading, its location and timing do not suggest that it arises because of a resource that is shared between syntactic and arithmetical operations. The eye fixations also suggest that subjects in this complex dual task engage in some processes, or scheduling of processes, that differ from those that occur when sentences are read under normal viewing conditions. All these results raise doubts about the inference Fedorenko et al. (2007) drew from their data that syntactic and arithmetical operations utilize a common pool of resources.

Acknowledgments We thank Evelina Fedorenko and Ted Gibson for sharing their data and materials and for comments on an earlier version of this work. This research was supported by Grant AG09661 to Gloria Waters.

\section{References}

Baayen, R. H., Davidson, D. J., \& Bates, D. M. (2008). Mixed-effects modeling with crossed random effects for subjects and items. Journal of Memory and Language, 59, 390-412.

Brysbaert, M., \& New, B. (2009). Moving beyond Kučera and Francis: A critical evaluation of current word frequency norms and the introduction of a new and improved word frequency measure for American English. Behavior Research Methods, 41, 977-990.

Caplan, D., \& Waters, G. S. (1999). Verbal working memory and sentence comprehension. Brain and Behavioral Sciences, 22, 77-126.

Fedorenko, E., Gibson, E., \& Rohde, D. (2007). The nature of working memory in linguistic, arithmetic and spatial integration processes. Journal of Memory and Language, 56, 246-269.

Jaeger, T. F. (2008). Categorical data analysis: Away from ANOVAs (transformation or not) and towards logit mixed models. Journal of Memory and Language, 59, 434-446.

King, J., \& Just, M. A. (1991). Individual differences in syntactic processing: The role of working memory. Journal of Memory and Language, 30, 580-602.

Lenth, R. V. (2009). Java Applets for Power and Sample Size [Computer software]. Retrieved August 4, 2011, from http:// www.stat.uiowa.edu/ rlenth/Power

Levy, R. (2008). Expectation-based syntactic comprehension. Cognition, 106, 1126-1177.

Staub, A. (2010). Eye movements and processing difficulty in object relative clauses. Cognition, 116, 71-86.

Sternberg, S. (1969). Memory-scanning: Mental processes revealed by reaction-time experiments. American Scientist, 57, 421-457.

Traxler, M. J., Morris, R. K., \& Seely, R. E. (2002). Processing subject and object-relative clauses: Evidence from eye-movements. Journal of Memory and Language, 47, 69-90.

Traxler, M. J., Williams, R. S., Blozis, S. A., \& Morris, R. K. (2005). Working memory, animacy, and verb class in the processing of relative clauses. Journal of Memory and Language, 53, 204-224.

Waters, G. S., Caplan, D., \& Rochon, E. (1995). Processing capacity and sentence comprehension in patients with Alzheimer's disease. Cognitive Neuropsychology, 12, 1-38. 